\title{
Tim-3 regulates the ability of macrophages to counter lipopolysaccharide-induced pulmonary epithelial barrier dysfunction via the PI3K/Akt pathway in epithelial cells
}

\author{
YUNTAO ZHANG $^{1 *}$ and WANG ZHANG ${ }^{2 *}$ \\ ${ }^{1}$ Department of Critical Care Medicine, The First Affiliated Hospital; ${ }^{2}$ Department of Infectious Diseases, \\ Sir Run Run Shaw Hospital, College of Medicine, Zhejiang University, Hangzhou, Zhejiang 310003, P.R. China
}

Received August 10, 2019; Accepted April 6, 2020

DOI: $10.3892 / \mathrm{mmr} .2020 .11109$

\begin{abstract}
Pulmonary epithelial barrier dysfunction is a critical pathological component of lung injury, caused primarily by impaired epithelial cell migration. Moreover, macrophage-epithelial interactions in pulmonary alveoli may either protect or damage epithelial barrier function. To investigate the effects of different macrophage subtypes, M1 and M2, on lipopolysaccharide (LPS)-induced epithelial barrier dysfunction, M1 and M2 macrophages were used to treat LPS-injured musculus lung epithelial cells (MLE-12). Barrier function was evaluated by monitoring cell monolayer permeability, T-cell immunoglobulin mucin 3 (Tim-3) small interfering RNA and anti-mouse Tim-3 antibody were used to knockdown or block endogenous Tim-3, to verify the role of the Tim-3 in macrophage-mediated barrier protection in LPS-injured MLE-12 cells. LY294002 was used to inhibit the activity of PI3K to verify the role of the PI3K/Akt signaling pathway in the restoration of epithelial cell. The present results revealed that co-culture of LPS-treated epithelial MLE-12 cells with M1 macrophages decreased cell migration and promoted permeability, whereas co-culture with M2 macrophages caused the opposite effects. It was determined that blocking T-cell immunoglobulin mucin 3 (Tim-3) signaling in macrophages and PI3K/Akt signaling in epithelial cells eliminated the barrier protection supplied by M2 macrophages. Tim-3, which maintains macrophage M2 polarization, is a key component of the macrophage-mediated barrier-repair process, while M2 macrophages regulate PI3K/Akt signaling in epithelial cells,
\end{abstract}

Correspondence to: Dr Yuntao Zhang, Department of Critical Care Medicine, The First Affiliated Hospital, College of Medicine, Zhejiang University, 79 Qingchun Road, Hangzhou, Zhejiang 310003, P.R. China

E-mail: zyticu@zju.edu.cn

*Contributed equally

Key words: T-cell immunoglobulin mucin 3, macrophages, polarization, epithelial injury, barrier dysfunction which in turn enhances pulmonary epithelial barrier function by restoring cell migration.

\section{Introduction}

Pulmonary epithelial cells are key regulators of lung homeostasis, providing a large surface area for gas exchange (1). Moreover, the physical barrier formed by the alveolar epithelium protects the body from damage caused by inhaled external materials, such as pathogenic microorganisms, and it also regulates the transport of water and ions (2). Pulmonary epithelial cells are particularly vulnerable to acute lung injury (ALI)/acute respiratory distress syndrome (ARDS) (3). Pulmonary epithelial barrier function is critically dependent on barrier integrity; the cells must engage in self-repair and self-renewal, and interact with various other cells in the context of pulmonary inflammation (4). Previous studies have revealed the potential relationships between dynamic cytoskeletal damage and epithelial barrier dysfunction, suggesting that cytoskeletal re-arrangement plays an important role in the development of lipopolysaccharide (LPS)-induced epithelial hyper permeability (5-7). Our previous study of the pulmonary microvascular endothelial barrier revealed that LPS modulated endothelial barrier function by regulating the dynamics of the cytoskeleton and intercellular junctions via the PI3K/Akt signaling pathway, which is a skeletal system-related pathway (8).

Effective pulmonary epithelial barrier repair is essential for preventing pathogen invasion and ALI/ARDS (1). In an ARDS animal model, inflammatory mediators such as interleukin (IL)-1 $\beta$, which triggers alveolar edema, mediate alveolar epithelial repair via a cytokine-dependent pathway involving epidermal growth factor and transforming growth factor- $\alpha$ (9). Furthermore, keratinocyte and hepatocyte growth factors in the pulmonary edema fluid of patients with ALI/ARDS stimulate alveolar epithelial repair in vitro (9). Several cells in the pulmonary inflammatory microenvironment, including endothelial cells and innate immune cells, also exert specific effects on the pulmonary epithelial barrier (10). Alveolar macrophages resident in the bronchi and alveoli respond immediately to harmful materials such as pathogens that reach the lower respiratory tract, as do alveolar epithelial cells (11). Macrophages can become polarized to one of two subtypes, 
M1 and M2, in several situations. M1 macrophages usually promote host defenses against bacteria or viruses and cause tissue damage, while M2 macrophages exhibit anti-inflammatory functions and promote tissue repair $(10,12)$. Moreover, M2 macrophages contribute to lung epithelial repair in patients with bacterial pneumonia, ventilator-associated lung injury and ALI/ARDS, but the specific mechanisms remain unknown (12). Our previous studies revealed that T-cell immunoglobulin mucin 3 (Tim-3), an immunomodulatory molecule, plays an important role in the mononuclear cell/macrophage system of patients with severe sepsis (13), and that Tim-3 may be involved in macrophage polarization (14). Therefore, the present study investigated whether there was a correlation between Tim-3 expression by macrophages and the regulation of alveolar pulmonary barrier function, and also whether barrier protection was induced by activation of a signaling cascade in epithelial cells.

\section{Materials and methods}

Cell isolation and culture. Murine bone marrow-derived macrophages (BMDMs) were generated from the femurs of male adult C57BL/6J mice [age, 6-7 weeks; wild-type; weight, 20-25 g; housed in a pathogen-free area at a controlled temperature $\left(20-26^{\circ} \mathrm{C}\right)$ with humidity $(40-60 \%)$ and free to access to food and water; Shanghai Model Organisms Biotech Co., Ltd.] as described previously (14). In brief, ten mice were anesthetized by the intraperitoneal injection of pentobarbital sodium $(1 \% ; 50 \mathrm{mg} / \mathrm{kg})$ and sacrificed by cervical dislocation; mortality was verified by observing the indications of breathing, heartbeat, pupil and nerve reflex. Bone marrow was flushed from the femurs, then bone marrow cell pellets were collected and treated with $10 \%(\mathrm{v} / \mathrm{v})$ red blood cell lysis buffer (v/v) on ice for $10 \mathrm{~min}$. Cell pellets were centrifuged at $1,500 \mathrm{x} \mathrm{g}$ at $4^{\circ} \mathrm{C}$ for $10 \mathrm{~min}$ and resuspended in complete medium [DMEM (Hyclone; Cytiva) containing 10\% FBS (cat. no. 0025; ScienCell Research Laboratories, Inc.) and $10 \mathrm{ng} / \mathrm{ml}$ macrophage colony-stimulating factor (cat. no. 315-02; PeproTech, Inc.)]. Cells were incubated at $37^{\circ} \mathrm{C}$ and $5 \% \mathrm{CO}_{2}$ for 3 days, and on day 4 half of the medium was replaced with new complete DMEM. After 7 days in culture, adherent cells were used in subsequent experiments. All animal studies were approved by the Animal Care and Use Committee of Zhejiang University and conformed to the Guide for the Care and Use of Laboratory Animals published by the US National Institutes of Health (NIH Publication no. 85-23; revised 1996).

The mouse peritoneal macrophage cell (RAW 264.7) and mouse lung epithelial cell (MLE-12) lines were obtained from the American Type Culture Collection; mycoplasma testing was performed for the cell lines. Cells were incubated in complete medium (DMEM with $10 \% \mathrm{FBS}$ ) at $37^{\circ} \mathrm{C}$ and $5 \%$ $\mathrm{CO}_{2}$.

Co-culture of macrophages and epithelial cells. Macrophages (RAW 264.7 and BMDMs) were differentiated to the M1 or M2 subtype using methods previously described (15). RAW 264.7 and BMDMs were differentiated into M1-polarized or M2-polarized macrophages by the addition of LPS (100 ng/ml; cat. no. L2880; Sigma-Aldrich; Merck KGaA) or IL-4 and
IL-13 (10 ng/ml each; PeproTech, Inc.) at $37^{\circ} \mathrm{C}$ with $5 \% \mathrm{CO}_{2}$ for $48 \mathrm{~h}$.

Transwell inserts (pore size, $0.4 \mu \mathrm{m}$; cat. no. 3450; Costar; Corning, Inc.) were used for macrophages and MLE-12 co-culture. In total, $1 \times 10^{6} \mathrm{M} 1$ or M2 macrophages (RAW 264.7 and BMDMs) were seeded into the upper chamber with DMEM. In the lower chamber, $2 \times 10^{6}$ MLE-12 cells were seeded and treated with or without LPS $(10 \mu \mathrm{g} / \mathrm{ml})$ in complete DMEM at $37^{\circ} \mathrm{C}$ for $24 \mathrm{~h}$. For PI3K inhibition, $25 \mu \mathrm{mol} / 1$ LY294002 (cat. no. 9901; Cell Signaling Technology, Inc.), which had no significant effect on untreated MLE-12 cells (Fig. S1A-E), was added to LPS (10 $\mu \mathrm{g} / \mathrm{ml})$-treated MLE-12 cells and incubated in serum-free and growth factor-free medium at $37^{\circ} \mathrm{C}$ and $5 \% \mathrm{CO}_{2}$ for $24 \mathrm{~h}$.

Flow cytometry. Adherent BMDMs were extracted as previously described (14). After cells were detached and washed using PBS, $0.5 \mu \mathrm{g}$ phycoerythrin-conjugated anti-mouse F4/80 (cat. no. 70-AM048004; MultiSciences Biotech Co., Ltd.) and $0.4 \mu \mathrm{g}$ allophycocyanin (APC)-conjugated anti-Mouse CD86 (cat. no. 70-AM08605; MultiSciences Biotech Co., Ltd.) were added to each tube. After incubation at room temperature for $30 \mathrm{~min}$, washing with PBS, and fixing and permeabilizing for 15 min with the FIX\&PERM kit (cat. no. 70-GAS003; MultiSciences Biotech Co.,Ltd.), BMDMs were incubated with $0.5 \mu \mathrm{g}$ APC-conjugated anti-Mouse CD206 (cat. no. 141707; BioLegend, Inc.) for $1 \mathrm{~h}$ at room temperature. BMDMs were then washed and analyzed by flow cytometry (BD FACS Calibur; BD Biosciences).

Immunofluorescence. RAW 264.7 and BMDMs were treated as previously described (8). After washing with PBS and fixing with $4 \%(\mathrm{w} / \mathrm{v})$ paraformaldehyde solution at room temperature for $20 \mathrm{~min}$, cells were incubated with Tim-3 antibody (1:500; cat. no. 60355-1-Ig; ProteinTech Group, Inc.) overnight at $4^{\circ} \mathrm{C}$. After washing three times with PBS, the cells were incubated with CoraLite594-conjugated antibody (1:200; cat. no. SA00013-3; ProteinTech Group, Inc.) at room temperature for $1 \mathrm{~h}$. Cells were washed with PBS and incubated with DAPI ( $1 \mu \mathrm{g} / \mathrm{ml}$; cat. no. D9564; Sigma-Aldrich; Merck KGaA) at room temperature for $10 \mathrm{~min}$. Then, the cells were washed in PBS and observed under a fluorescent microscope (magnification, x400; Leica Microsystems).

Reverse transcription-quantitative PCR (RT-qPCR). Total RNA was extracted from cells and RT to cDNA was performed by using the RNA Extraction kit (cat. no. 9767; Takara Bio, Inc.) and the PrimeScript RT Master Mix (cat. no. RR036A; Takara Bio, Inc.) as previously described (14). Subsequently, qPCR was performed using SYBR RT-qPCR Master mix (cat. no. Q311-02-AA; Vazyme Biotech Co., Ltd.). The following thermocycling conditions were used for qPCR: Initial denaturation at $95^{\circ} \mathrm{C}$ for $30 \mathrm{sec}$, followed by 40 cycles at $95^{\circ} \mathrm{C}$ for $5 \mathrm{sec}$ and $60^{\circ} \mathrm{C}$ for $30 \mathrm{sec}$. mRNA expression levels were quantified using the $2^{-\Delta \Delta \mathrm{Cq}}$ method (16). Sequences of the primers used in this study are presented in Table SI.

Small interfering RNA (siRNA) transfections and Tim-3 blockade. Negative control siRNA and Tim-3-specific siRNA (forward, CCUAACCACGGAGAGAAAUTT 
and reverse, AUUUCUCUCCGUGGUUAGGGT; Gene Chemical Technology Co., Ltd.) were transfected (30 pmol) into RAW 264.7 cells ( $1 \times 10^{5}$ cells) using INTERFERin (cat. no. 409-10; Polyplus-transfection SA) for $24 \mathrm{~h}$ before subsequent experimentation. BMDMs $\left(1 \times 10^{6}\right)$ were treated with $10 \mu \mathrm{g} / \mathrm{ml}$ anti-mouse Tim-3-blocking monoclonal $\mathrm{Ab}$ (mAb; cat. no. 134002 ; BioLegend, Inc.) at $37^{\circ} \mathrm{C}$ for $48 \mathrm{~h}$ for function blocking.

Transwell-Evans blue monolayer permeability assay. Transwell inserts (pore size, 3.0- $\mu \mathrm{m}$; cat. no. 3472; Costar; Corning, Inc.) were used to evaluate permeability as previously described (8). In brief, $1 \times 10^{5}$ MLE-12 cells were seeded in inserts and treated with LPS $(10 \mu \mathrm{g} / \mathrm{ml})$ at $37^{\circ} \mathrm{C}$ for $24 \mathrm{~h}$, then co-cultured with RAW 264.7 or BMDMs $\left(1 \times 10^{6}\right)$. Subsequently, $100 \mu$ l Evans Blue (EB)-conjugated albumin $(0.67 \mathrm{mg} / \mathrm{ml}$; Sigma-Aldrich; Merck KGaA) was added to the upper chamber as previously described (17). To the lower chamber, $500 \mu 1$ 4\% BSA (Sigma-Aldrich; Merck KGaA) was added. After incubation for $1 \mathrm{~h}$ at $37^{\circ} \mathrm{C}$, the medium in the lower chambers was collected. The absorbance was determined at a wavelength of $620 \mathrm{~nm}$ using a micro-plate reader. The trans-epithelial cell EB-albumin leak was calculated according to standard curve.

Wound healing assay. Based on a previous description (8), $2 \times 10^{6}$ MLE-12 cells were seeded in 6-well plates. Confluent monolayer cells were scraped by using a 1,000 $\mu \mathrm{l}$ pipette tip and then washed with PBS. MLE-12 cells were treated with LPS $(10 \mu \mathrm{g} / \mathrm{ml})$ at $37^{\circ} \mathrm{C}$ for $24 \mathrm{~h}$, then co-cultured in DMEM (with $2 \%$ FBS) with RAW 264.7 or BMDMs. The images were captured using a light microscope at 0 and $24 \mathrm{~h}$ at the same position of the wound. The migratory ability was assessed by the rate of scratch wound confluence using Adobe Photoshop 2017 software (Adobe Systems, Inc.) and the following formula: $\%$ wound confluence $=(a-b) \times 100 \% / a$, where a is the initial scratch wound area at $0 \mathrm{~h}$ and $\mathrm{b}$ is the scratch wound area at $24 \mathrm{~h}$.

Western blotting. Total protein was extracted from MLE-12 cells using RIPA buffer (Beyotime Institute of Biotechnology) and quantified using the BCA kit (Beyotime Institute of Biotechnology), as previously described (14). Protein samples (10 $\mu \mathrm{g}$ per lane) were separated via $10 \%$ SDS-PAGE and transferred to PVDF membranes. The membranes were then blocked with $5 \%(\mathrm{w} / \mathrm{v})$ skimmed milk at room temperature for $1 \mathrm{~h}$. Then, the membranes were incubated with primary antibodies at $4^{\circ} \mathrm{C}$ overnight, including phosphorylated (p)-Akt (1:2,000; cat. no. 4060; Cell Signaling Technology, Inc.) and Akt (1:1,000; cat. no. 4685; Cell Signaling Technology, Inc.). The membranes were incubated with a horseradish peroxidase-conjugated secondary antibody (1:2,000; cat. no. A0208; Beyotime Institute of Biotechnology) and developed with an enhanced chemiluminescence kit (cat. no. 70-P1421; Multi Sciences Biotech) and exposed to X-ray film. Protein expression was semi-quantified using Image $\mathrm{J}$ software (version 1.8.0, National Institutes of Health).

Statistical analysis. All experiments were repeated at least three times. Data are presented as the mean $\pm \mathrm{SD}$. A two-tailed
Student's t-test was used for two-group comparisons and one-way ANOVA followed by Tukey's multiple comparisons test was used for multiple-group comparisons. GraphPad Prism 7.0 (GraphPad Software, Inc.) was used for analysis. $\mathrm{P}<0.05$ was considered to indicate a statistically significant difference.

\section{Results}

Co-culture with macrophages affects the migration and monolayer permeability of LPS-treated MLE-12 cells. To identify and quantify the number of M1/M2 cells present after various standard treatments, the expression levels of M1/M2 markers were assessed in RAW 264.7 cells and BMDMs using both RT-qPCR and flow cytometry. The mRNA expression of the M1 marker inducible nitric oxide synthase (iNOS) in RAW 264.7 cells and the number of $\mathrm{F} 4 / 80^{+} \mathrm{CD} 86^{+} \mathrm{BMDMs}$ were significantly upregulated by LPS stimulation, thus indicating successful M1 polarization. Moreover, it was revealed that mRNA expression of the M2 marker Arginase 1 (Arg-1) in RAW 264.7 cells and the number of $\mathrm{F} 4 / 80^{+} \mathrm{CD} 206^{+} \mathrm{BMDMs}$ were significantly upregulated by IL-4 and IL-13 stimulation, indicating successful M2 polarization (Fig. 1A and B). In addition, LPS-pretreated MLE-12 cells upregulated the expression levels of M1 markers, but did not downregulate the expression levels of M2 markers in M2 macrophages compared with untreated M2 macrophages (Fig. S1G).

To determine the effects of different macrophage subtypes (RAW 264.7 and BMDMs) on MLE-12 cell migration after treatment with LPS, migratory rates were measured using a wound healing assay. The extent of scratch wound confluence at $24 \mathrm{~h}$ was significantly decreased in the LPS group compared with the control and M2 macrophage co-culture groups, and also significantly reduced in the M1 macrophage co-culture group compared with the control group (Fig. 1C-F). However, no significant difference was demonstrated between the LPS and M1 macrophage co-culture groups. The results revealed that EB leakage from the upper chamber, reflecting monolayer permeability, was significantly increased in the LPS group compared with the control and M2 macrophage co-culture groups, and was also significantly greater in the M1 macrophage (BMDMs) co-culture group compared with the LPS group (Fig. 2A).

Tim-3 expression in different subtypes of macrophages. To assess Tim- 3 expression in different subtypes of macrophages, RT-qPCR and immunofluorescence labeling were used to quantify Tim-3 expression in M1/M2 macrophages. RT-qPCR results indicated that the mRNA expression of Tim-3 was significantly increased in M2 macrophages (RAW 264.7 and BMDMs); however, the expression in the control and M1 macrophage groups was not significantly different (Fig. 2B and C). Furthermore, it was revealed that Tim-3 fluorescence intensity exhibited a similar trend, as it was increased in M2 but decreased in M1 macrophages (Fig. 2D).

Tim-3 blockade or knockdown in M2 macrophages abolishes the restoration of the migratory ability and monolayer permeability in LPS-treated MLE-12 cells. To investigate whether co-culture with M2 macrophages modulated the lung 

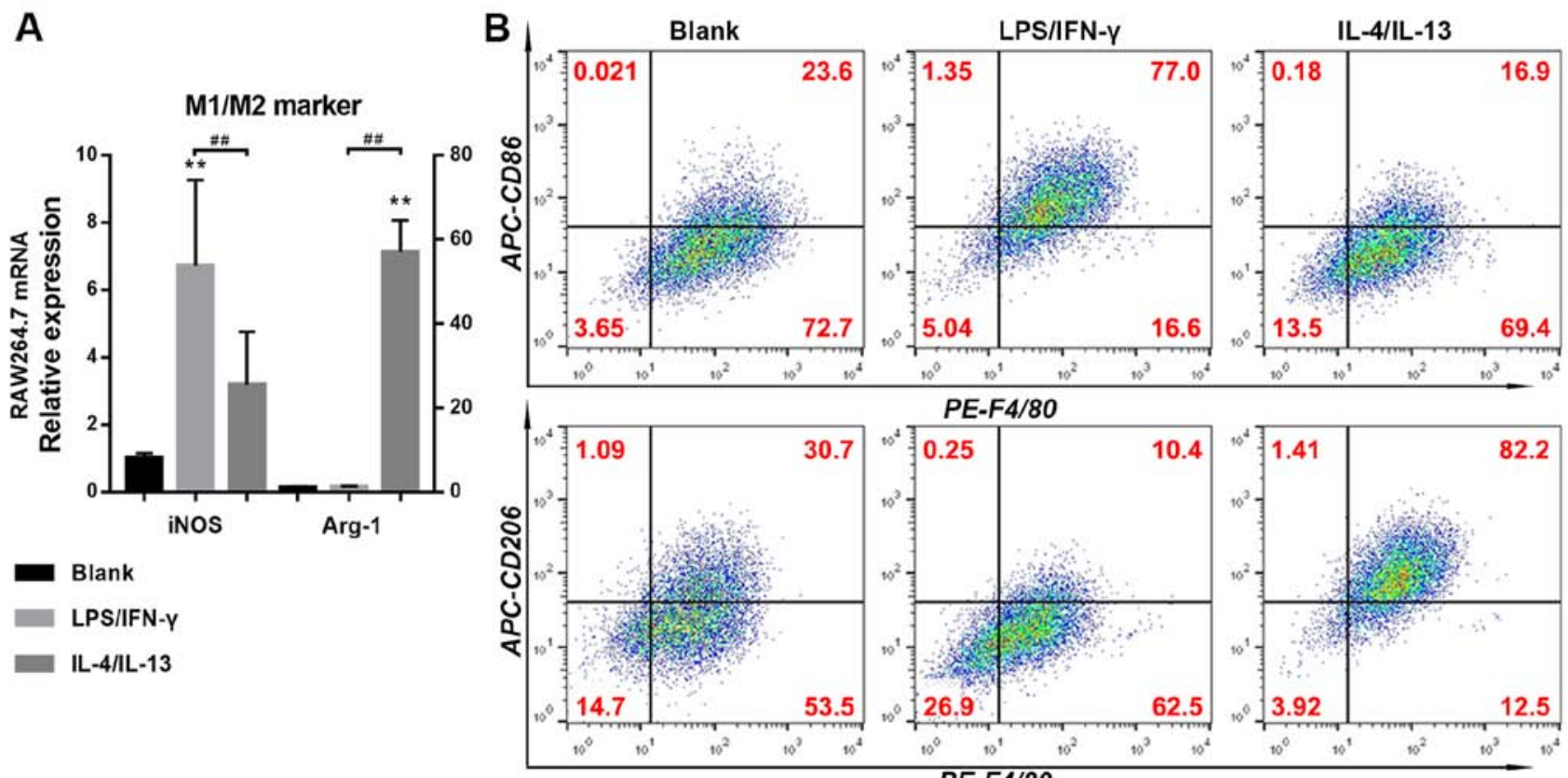

C

RAW264.7
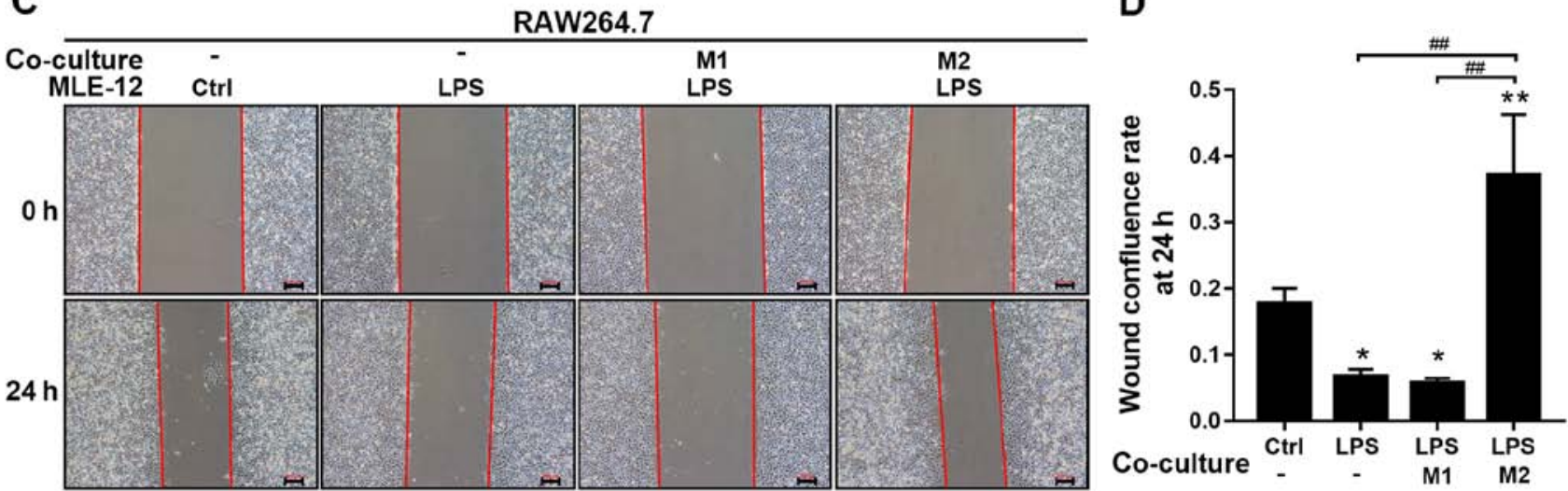

E

BMDMs
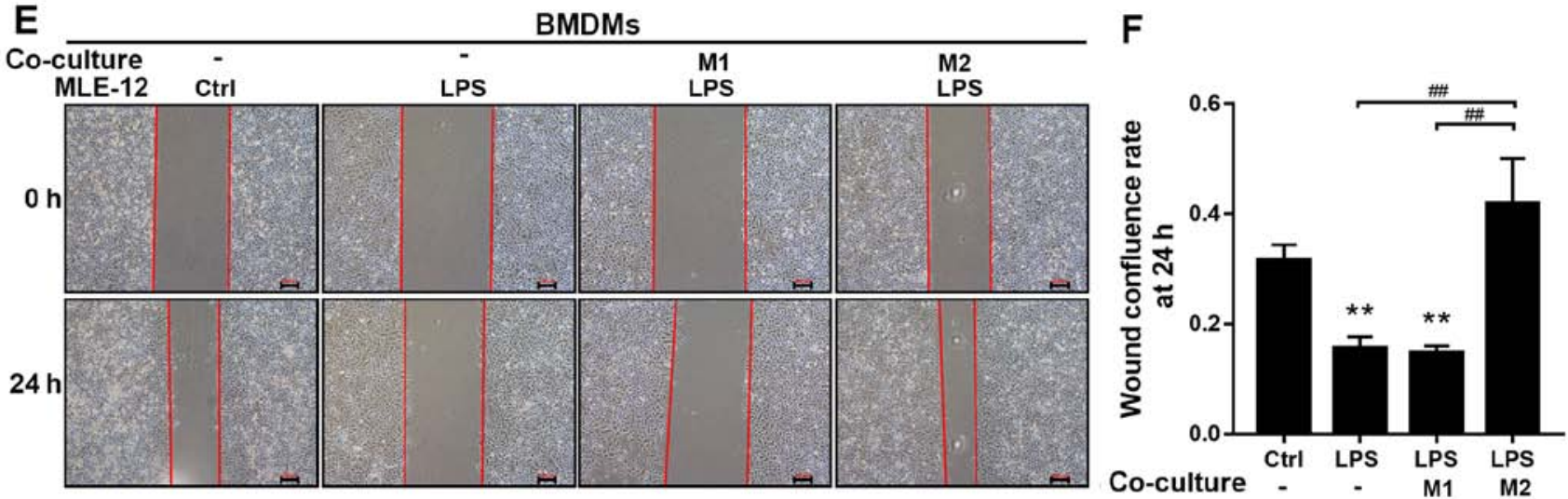

Figure 1. Effects of M1 and M2 macrophages on the migration of LPS-treated MLE-12 cells. (A) mRNA expression levels of the M1 marker iNOS and the M2 marker Arg-1 in RAW 264.7 cells were measured using reverse transcription-quantitative PCR. (B) Proportions of M1 subtype (F4/80 $\left.{ }^{+} \mathrm{CD} 86^{+}\right)$and M2 subtype macrophages $\left(\mathrm{F} 4 / 80^{+} \mathrm{CD} 206^{+}\right)$were examined by flow cytometry. (C) Wound healing assay was used to assess the (D) migration of MLE-12 cells co-cultured with M1/M2-like RAW 264.7 after treatment with LPS. (E) Wound healing assay was used to assess the (F) migration of MLE-12 cells co-cultured with M1/M2-like BMDMs after treatment with LPS.Quantification of the confluence rate at $24 \mathrm{~h}$, which represents the migration ability [\% wound confluence $=$ (a - b) x 100\% / a, where a represents the initial scratch wound area at $0 \mathrm{~h}$ and b represents the scratch wound area at $24 \mathrm{~h}]$. Scale bars, $200 \mu \mathrm{m}$. Data are from three independent experiments and presented as the mean $\pm \mathrm{SD} .{ }^{*} \mathrm{P}<0.05,{ }^{* * *} \mathrm{P}<0.01$ vs. the control group. ${ }^{\# \prime} \mathrm{P}<0.01$. iNOS, inducible nitric oxide synthase; Arg- 1 , Arginase 1; LPS, lipopolysaccharide; BMDM, bone marrow-derived macrophages; IL, interleukin.

epithelial barrier function mediated by Tim-3, RAW 264.7 cells were transfected with Tim-3 siRNA, and knockdown efficiency was $\sim 70 \%$ (Fig. S1F; Fig. 3A). It was demonstrated that knockdown of endogenous Tim-3 significantly compromised the barrier protection induced by co-culture of M2-polarized RAW 264.7 cells (Fig. 3F). Moreover, Tim-3 siRNA 
A

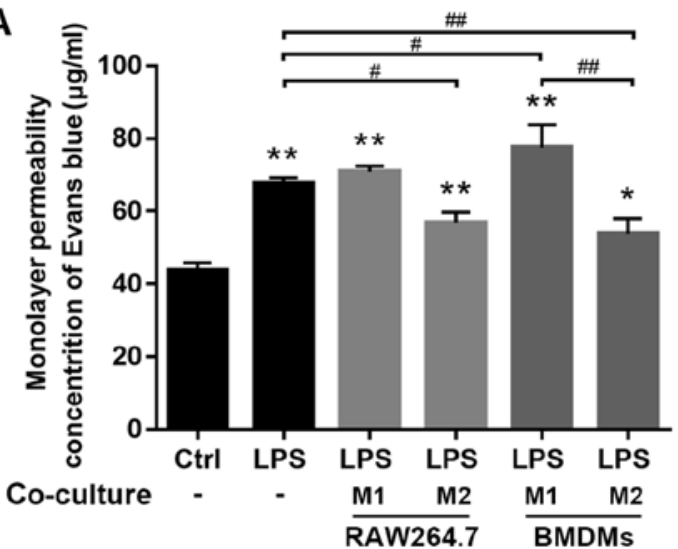

B

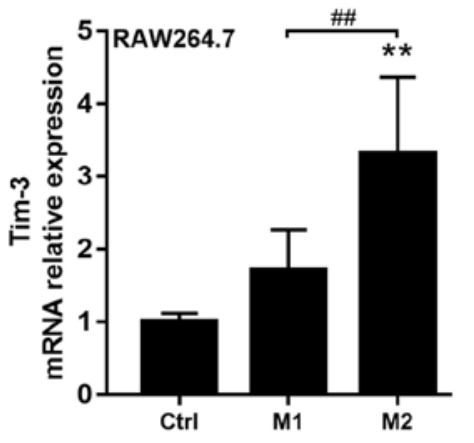

C

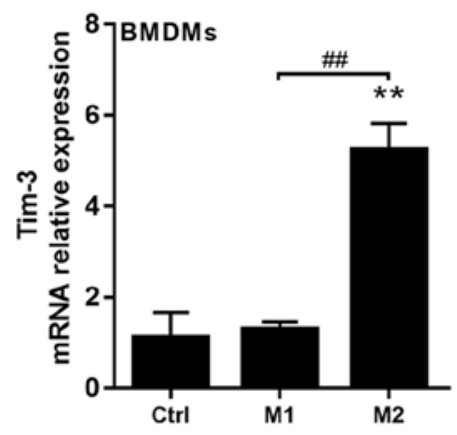

D

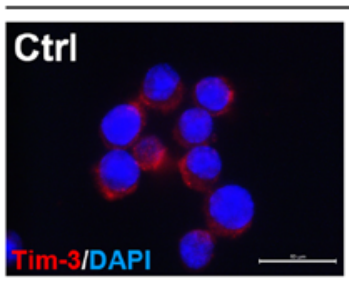

RAW264.7
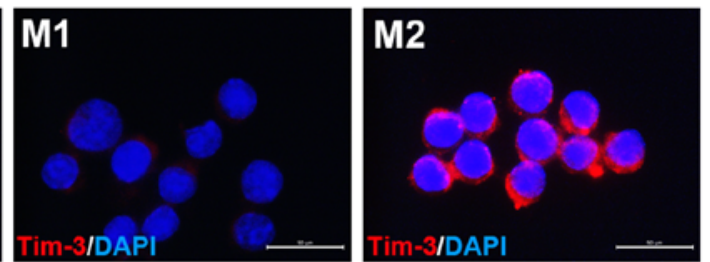

BMDMs
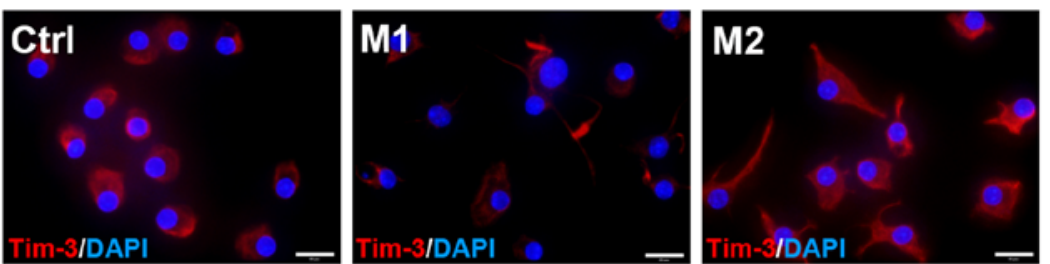

Figure 2. Effects of different macrophage subtypes on permeability of LPS-treated MLE-12 cells and Tim-3 expression. (A) Monolayer permeability was measured using a Transwell-EB assay. EB concentrations in the lower chambers of each group represented monolayer permeability. mRNA expression of Tim-3 in (B) M1/M2-like RAW 264.7 cells and (C) BMDMs. (D) Expression of Tim-3 protein was evaluated by immunofluorescence assay. Red indicates Tim-3 and blue indicates nuclei. Scale bars, $50 \mu \mathrm{m} .{ }^{*} \mathrm{P}<0.05,{ }^{* *} \mathrm{P}<0.01$ vs. the control group. ${ }^{\#} \mathrm{P}<0.05$, ${ }^{\#} \mathrm{P}<0.01$. EB, Evans Blue; LPS, lipopolysaccharide; Tim-3, T-cell immunoglobulin mucin 3; BMDM, bone marrow-derived macrophages; Ctrl, control.

significantly inhibited the promoting effect of M2-polarized RAW 264.7 cells on MLE-12 cell migratory ability, which was inhibited by LPS (Fig. 3B and C). It was also determined that the extent of EB leakage from the upper chamber was significantly increased in the M2 + Tim-3 siRNA co-culture group compared with the M2 and M2 + negative control co-culture groups (Fig. 3F). Similar effects were observed when M2-polarized BMDMs were incubated with a Tim-3-blocking $\mathrm{mAb}$; the extent of MLE-12 cell confluence at $24 \mathrm{~h}$ was significantly reduced in the $\mathrm{M} 2+\mathrm{Tim}-3 \mathrm{mAb}$ co-culture group compared with the M2 co-culture group (Fig. 3D and E). The results indicated that $\mathrm{EB}$ leakage from the upper chamber was significantly increased in the M2 + Tim-3 mAb co-culture group compared with the M2 co-culture group (Fig. 3G).

Next, the present study assessed M1/M2 marker expression levels in M2 macrophages after Tim-3 signaling was reduced or blocked. mRNA expression of the M1 marker iNOS in RAW 264.7 cells and the number of F4/80 ${ }^{+} \mathrm{CD}^{+}$ BMDMs were significantly upregulated in the presence of Tim-3 siRNA or Tim-3 mAb. However, the mRNA expression of the M2 marker Arg-1 in RAW 264.7 cells and the number of $\mathrm{F} 4 / 80^{+} \mathrm{CD} 206^{+} \mathrm{BMDMs}$ were significantly downregulated by Tim-3 siRNA or Tim-3 mAb (Fig. 3H and I).
M2 macrophages restore epithelial barrier via the PI3k/Akt signaling pathway in epithelial cells. Western blotting was used to assess the ratio of p-Akt to total Akt expression. The ratio of p-Akt to total Akt was significantly higher in MLE-12 cells co-cultured with M2 macrophages (BMDMs) compared with cells exposed to LPS alone and cells co-cultured with M1 macrophages (Fig. 4A and B). The present study also examined whether the PI3K/Akt pathway in MLE-12 cells was involved in the barrier protection provided by co-culture with M2 macrophages. LY294002 $(25 \mu \mathrm{M})$, a PI3K inhibitor, was used to assess whether inhibition of the PI3K/Akt pathway compromised the protective effect. It was determined that LY294002 inhibited the restoration of MLE-12 cell migration and monolayer permeability after co-culture with M2 macrophages (Fig. 4C-E). Furthermore, LY294002 reduced p-Akt protein expression (Fig. 4F and G).

\section{Discussion}

The respiratory system is often exposed to harmful substances from the airway or bloodstream (10). Moreover, a variety of lung cells are involved in the maintenance of pulmonary homeostasis; alveolar macrophages, epithelial cells and other 
A

A B

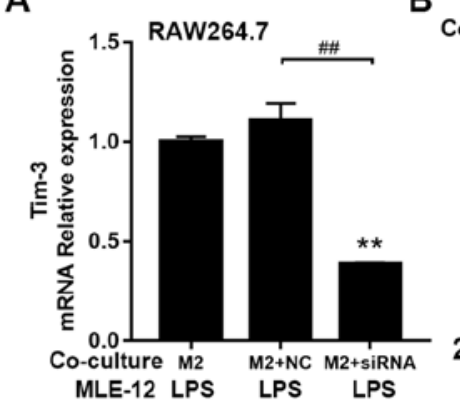

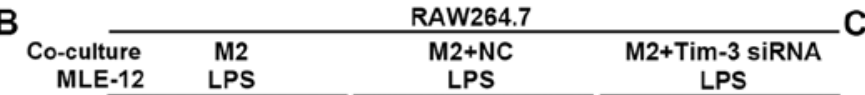

C

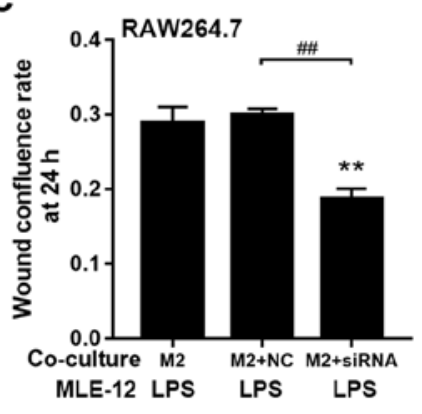

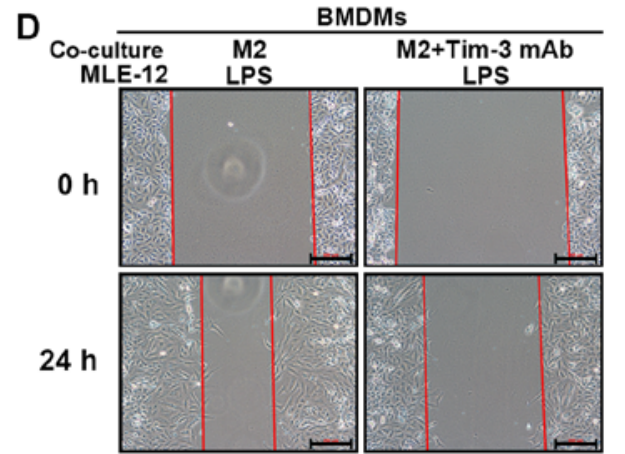

$\mathrm{H}$

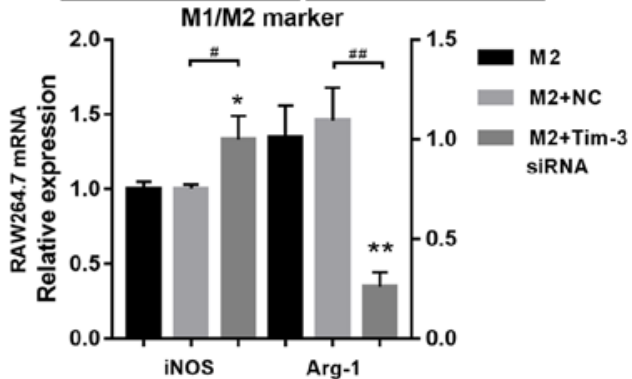

E

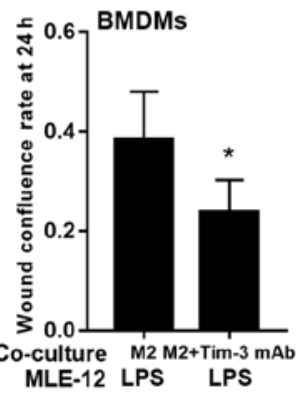

I
$\mathbf{F}$

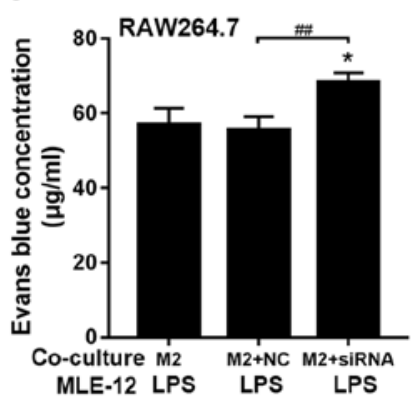

G

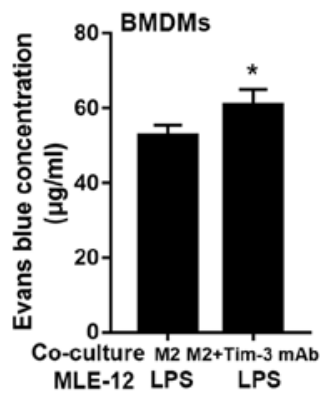

Figure 3. Effects of Tim-3 blockade on monolayer permeability and migration of LPS-injured MLE-12 cells co-cultured with M2 macrophages. (A) Tim-3-siRNA knockdown efficiency $(\sim 70 \%)$ was measured using reverse transcription-quantitative PCR. (B) Wound healing assay was used to assess the (C) migration of MLE-12 cells co-cultured with M2-like RAW 264.7 cells treated with Tim-3 specific siRNA. (D) Wound healing assay was used to assess the (E) migration of MLE-12 cells co-cultured with M2-like BMDMs treated with Tim-3 mAb. EB leakage from MLE-12 monolayers co-cultured with (F) Tim-3-knockdown M2-like RAW 264.7 cells and (G) Tim-3 mAb-treated M2-like BMDMs. (H) mRNA expression levels of the M1 marker iNOS and the M2 marker Arg-1 in Tim-3-knockdown M2-like RAW 264.7 cells. (I) Proportions of M1 subtype (F4/80+CD86 ${ }^{+}$) and M2 subtype macrophages (F4/80+CD206 ${ }^{+}$in Tim-3 mAb-treated M2-like BMDMs. Scale bar, $200 \mu \mathrm{m}$. ${ }^{*} \mathrm{P}<0.05,{ }^{* *} \mathrm{P}<0.01$ vs. the M2 co-culture group; ${ }^{\#} \mathrm{P}<0.05$, ${ }^{\# \#} \mathrm{P}<0.01$. EB, Evans Blue; LPS, lipopolysaccharide; Tim-3, T-cell immunoglobulin mucin 3; BMDM, bone marrow-derived macrophages; mAb, monoclonal antibody; iNOS, inducible nitric oxide synthase; Arg-1, Arginase 1; siRNa, small interfering RNA; NC, negative control.

cells play important roles $(4,12)$. Host-pathogen interactions can injure pulmonary epithelial cells and trigger barrier dysfunction; thus, the alveolar space is no longer protected against fluid entry from adjacent vascular compartments (10). The resulting alveolar edema is termed ALI/ARDS, which is a syndrome characterized by intractable hypoxemia, respiratory distress, progressive respiratory failure and high mortality and morbidity rates (18). LPS, also known as endotoxins, is a pro-inflammatory component of the outer membrane of Gram-negative bacteria and plays an important role in infection (10). LPS is the principal pathogenic component of airway microorganisms that can cross the epithelial barrier to trigger sepsis-related barrier dysfunction, where it activates innate immune cells, triggers local and systemic inflammatory responses and disrupts barrier function; LPS is typically used to induce ALI/ARDS in animal models $(8,16)$. The present study used LPS to stimulate mouse lung epithelial cells to simulate the damage induced in the pulmonary epithelial barrier under inflammatory conditions. It was revealed that epithelial barrier permeability increased and cell migration decreased after LPS treatment.

Previous studies have revealed that pulmonary epithelial cells are involved in the maintenance of lung homeostasis, as epithelial cell secretions contain enzymes such as lysozyme that damage bacteria $(19,20)$. Furthermore, it has been revealed that pulmonary epithelial cells maintain humoral and ionic homeostasis by regulating the cytoskeleton, cell junctions and ion channels $(4,21)$. Destruction of the epithelial barrier can also trigger recurrent infections and ALI (1). It has been revealed that pulmonary epithelial barrier repair is useful for treating lung injury (22) and that certain cytokines may play important roles in injury repair, and thus are attracting increasing attention $(9,23)$. Numerous cellular interactions are involved in the alveolar inflammatory microenvironment; alveolar macrophages play important roles in pathogen recognition and recruitment of immune cells to the 
A
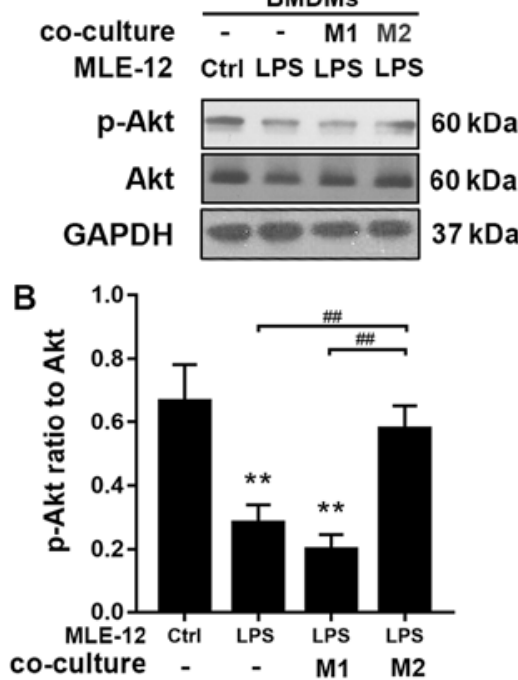

$\mathrm{E}$

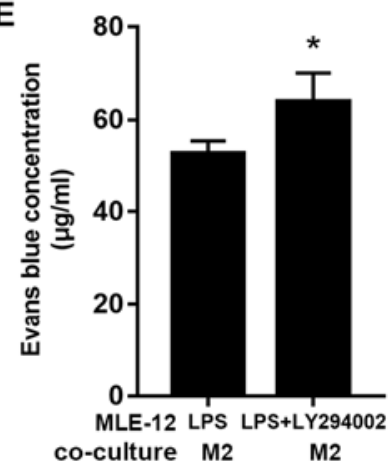

C

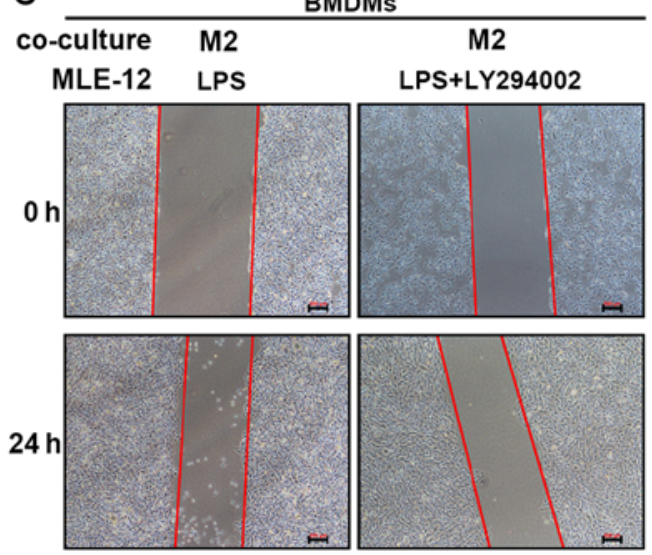

$\mathbf{F}$

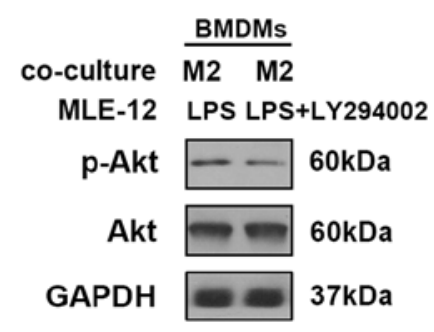

D

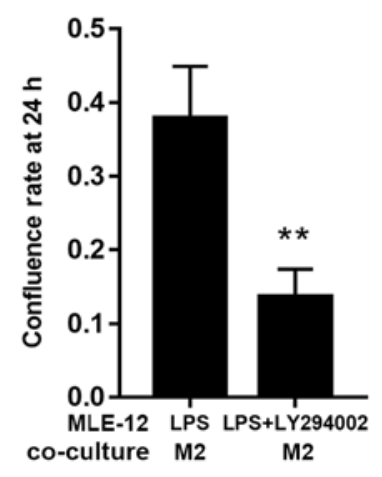

G

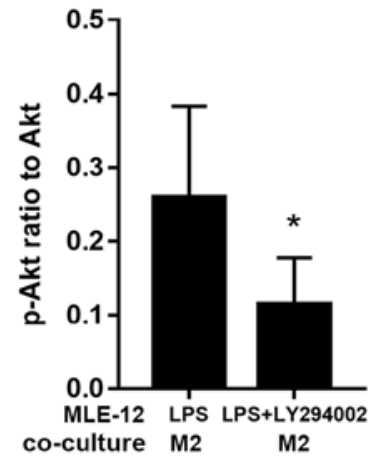

Figure 4. Effects of PI3K/Akt inhibition on monolayer permeability and migration of LPS-injured MLE-12 cells co-cultured with M2 macrophages. (A) Akt phosphorylation in MLE-12 cells co-cultured with M2-like BMDMs was assessed by western blotting. (B) Quantification of the ratio of p-Akt to total Akt. (C) Wound healing assay was used to assess the (D) migration of LY294002 pre-treated MLE-12 cells co-cultured with M2-like BMDMs. (E) Evans Blue leakage of LY294002 pre-treated MLE-12-monolayer co-cultured with M2-like BMDMs. (F) Western blotting was used to assess (G) the ratio of p-Akt to total Akt protein, which was significantly decreased in the LPS + LY294002 group compared with the LPS group of MLE-12 cells. Scale bar, $200 \mu \mathrm{m}$. "P<0.05, ${ }^{* *} \mathrm{P}<0.01$ vs. the M2 co-culture group. ${ }^{\#} \mathrm{P}<0.01$. p-, phosphorylated; LPS, lipopolysaccharide; BMDM, bone marrow-derived macrophages; Ctrl, control.

alveoli (4). Different macrophage subtypes inhibit inflammation via multiple pathways, including switching of M1 macrophages to the M2 subtype, induction of efferocytosis to clear neutrophils and secretion of anti-inflammatory mediators $(4,12)$, thus enhancing damage repair. A previous study revealed that adaptive transfer of M2 macrophages promotes lung injury repair and reduces pulmonary inflammation in mice; in contrast, M2 macrophages aggravate lung injury (24). In line with these previous results, the present results indicated that co-culture of epithelial cells with M1 macrophages aggravated the epithelial barrier permeability induced by LPS, whereas co-culture with M2 macrophages significantly attenuated LPS-induced epithelial leakage and upregulated the migration of epithelial cells. Therefore, the present study identified the important roles played by different macrophage subtypes in the induction and repair of pulmonary epithelial damage.

Tim-3 is an immunomodulatory molecule that is highly expressed in cytotoxic T and helper T1 (Th1) cells, which engages in inhibitory signaling that triggers apoptosis of cytotoxic T and helper T1 cells (25). Tim-3 is also expressed in monocytes, macrophages and dendritic cells, where it plays an important role in immune regulation (26). Furthermore, Tim-3 has been revealed to regulate the functions of monocytes and macrophages in both humans and mice; a previous study based on a sepsis model revealed that Tim-3 signaling reduced the release of inflammatory factors, inhibited macrophage function and provided pulmonary protection (27). Furthermore, it has been revealed that blockade of Tim-3 signaling increases sepsis severity and significantly reduces mouse survival (28). Our previous study using clinical samples revealed that Tim-3 expression was increased in $\mathrm{T}$ lymphocytes, but decreased in mononuclear cells and macrophages in patients with sepsis (13). In addition, upregulation of Tim-3 expression in $\mathrm{T}$ cells may be associated with T-cell apoptosis and functional depletion, but the function of Tim-3 in monocytes is not fully understood (13). Therefore, the present study investigated whether Tim-3 expression in M2 macrophages affected epithelial barrier function in a macrophage-epithelial cell co-culture model. It was demonstrated that Tim-3 was weakly expressed in M1 macrophages, but highly expressed in M2 macrophages; it was also determined that interference or blockade of Tim-3 in M2 macrophages compromised repair of the lung epithelial barrier, which was associated with macrophage polarization. Therefore, it was speculated that Tim-3 may be involved in macrophage M1/M2 switching. 
It is important to identify regulatory molecules expressed in epithelial cells that are associated with macrophages. The PI3K/Akt pathway not only regulates various cellular functions, but also has roles in the signaling underlying epithelial barrier function during sepsis and ALI/ARDS (29,30). The present study identified activated PI3K/Akt signaling in MLE-12 cells co-cultured with M2 macrophages; however, the pathway was inhibited by LPS. The PI3K inhibitor LY294002 was used in the present study to examine whether the PI3K/Akt pathway mediates M2 macrophage-induced barrier protection. It was demonstrated that inhibition of PI3K/Akt signaling suppressed cell migration and barrier restoration induced by M2 macrophage co-culture, suggesting that Tim-3 regulates the function of macrophages, which indirectly regulates PI3K/Akt activity in epithelial cells rather than macrophages, thus affecting the barrier function of epithelial cells. Based on the Transwell assay results, cell-cell direct contact may not be required for barrier protection by M2 macrophage; however, some M1/M2-produced cytokines or chemokines may be involved in the process. It has been reported that epithelial and innate immune cell interactions mediate wound healing and tissue restoration (31). Moreover, cytokines such as IL-10 (32) and extracellular vesicles (33) produced by macrophages participate in epithelial wound repair, and thus future studies will investigate whether Tim-3 mediates these processes in macrophages. In addition, MLE-12 in wound healing assay should be serum-starved, however, due to its intolerance to serum-free medium, $2 \%$ FBS was used, which was a limitation of this study.

In conclusion, it was revealed that macrophage Tim-3 affects macrophage polarization. Furthermore, the present results indicated that M1 macrophages promoted inflammation and aggravated pulmonary epithelial barrier dysfunction. However, M2 macrophages attenuated inflammation, thus promoting repair of the lung epithelial barrier after injury, and this function was speculated to be mediated by PI3K/Akt signaling in epithelial cells. Given the protective role of M2 macrophages, control of macrophage phenotype rather than complete macrophage depletion may be a potential therapeutic approach to treat injury repair. Furthermore, the present results suggested that Tim-3 may be a valuable therapeutic target.

\section{Acknowledgements}

Not applicable.

\section{Funding}

The present study was supported by Zhejiang Provincial Natural Science Foundation (grant no. LY16H150003).

\section{Availability of data and materials}

The datasets used and/or analyzed during the current study are available from the corresponding author on reasonable request.

\section{Authors' contributions}

YZ and WZ wrote the main manuscript text and performed the experiments. YZ also analyzed the data.

\section{Ethics approval and consent to participate}

All animal studies were approved by the Animal Care and Use Committee of Zhejiang University and conformed to the Guide for the Care and Use of Laboratory Animals published by the US National Institutes of Health (NIH Publication no. 85-23; revised 1996).

\section{Patient consent for publication}

Not applicable.

\section{Competing interests}

The authors declare that they have no competing interests.

\section{References}

1. Brune K, Frank J, Schwingshackl A, Finigan J and Sidhaye V: Pulmonary epithelial barrier function: Some new players and mechanisms. Am J Physiol Lung Cell Mol Physiol 308: L731-L745, 2015.

2. Gunther J and Seyfert HM: The first line of defence: Insights into mechanisms and relevance of phagocytosis in epithelial cells. Semin Immunopathol 40: 555-565, 2018.

3. Guillot L, Nathan N, Tabary O, Thouvenin G, Le Rouzic P, Corvol H, Amselem S and Clement A: Alveolar epithelial cells: Master regulators of lung homeostasis. Int J Biochem Cell Biol 45: 2568-2573, 2013.

4. Bhattacharya JK: Westphalen, Macrophage-epithelial interactions in pulmonary alveoli. Semin Immunopathol 38: 461-469, 2016.

5. Rodgers LS and Fanning AS: Regulation of epithelial permeability by the actin cytoskeleton. Cytoskeleton (Hoboken, N.J.) 68: 653-660, 2011.

6. Herold S, Gabrielli NM and Vadasz I: Novel concepts of acute lung injury and alveolar-capillary barrier dysfunction. Am J Physiol Lung Cell Mol Physiol 305: L665-L681, 2013.

7. Wang W, Weng J, Yu L, Huang Q, Jiang Y and Guo X: Role of TLR4-p38 MAPK-Hsp27 signal pathway in LPS-induced pulmonary epithelial hyperpermeability. BMC Pulm Med 18: 178, 2018.

8. Zheng X, Zhang W and Hu X: Different concentrations of lipopolysaccharide regulate barrier function through the PI3K/Akt signalling pathway in human pulmonary microvascular endothelial cells. Sci Rep 8: 9963, 2018.

9. Geiser T: Mechanisms of alveolar epithelial repair in acute lung injury-a translational approach. Swiss Med Wkly 133: 586-590, 2003 .

10. Nova Z, Skovierova $\mathrm{H}$ and Calkovska A: Alveolar-capillary membrane-related pulmonary cells as a target in endotoxin-induced acute lung injury. Int J Mol Sci 20: pii: E831, 2019.

11. Reynier F, de Vos AF, Hoogerwerf JJ, Bresser P, van der Zee JS, Paye M, Pachot A, Mougin B and van der Poll T: Gene expression profiles in alveolar macrophages induced by lipopolysaccharide in humans. Mol Med 18: 1303-1311, 2012.

12. Aggarwal NR, King LS and D'Alessio FR: Diverse macrophage populations mediate acute lung inflammation and resolution. Am J Physiol Lung Cell Mol Physiol 306: L709-L275, 2014.

13. Xia Q, Wei L, Zhang Y, Sheng J, Wu W and Zhang Y: Immune checkpoint receptors Tim-3 and PD-1 regulate monocyte and T Lymphocyte function in septic patients. Mediators Inflamm 2018: $1632902,2018$.

14. Zhang W,Zhang Y,He Y, Wang X and Fang Q: Lipopolysaccharide mediates time-dependent macrophage M1/M2 polarization through the Tim-3/Galectin-9 signalling pathway. Exp Cell Res 376: 124-132, 2019.

15. Hunter MM, Wang A, Parhar KS, Johnston MJ, Van Rooijen N, Beck PL and McKay DM: In vitro-derived alternatively activated macrophages reduce colonic inflammation in mice. Gastroenterology 138: 1395-1405, 2010.

16. Livak KJ and Schmittgen TD: Analysis of relative gene expression data using real-time quantitative PCR and the 2(-Delta Delta C(T)) method. Methods 25: 402-408, 2001. 
17. Patterson CE, Rhoades RA and Garcia JG: Evans blue dye as a marker of albumin clearance in cultured endothelial monolayer and isolated lung. J Appl Physiol (1985) 72: 865-873, 1992.

18. Rubenfeld GD, Caldwell E, Peabody E, Weaver J, Martin DP, Neff M, Stern EJ and Hudson LD: Incidence and outcomes of acute lung injury. N Engl J Med 353: 1685-1693, 2005.

19. Ellison RT III and Giehl TJ: Killing of gram-negative bacteria by lactoferrin and lysozyme. J Clin Invest 88: 1080-1091, 1991.

20. Ibrahim HR, Aoki T and Pellegrini A: Strategies for new antimicrobial proteins and peptides: Lysozyme and aprotinin as model molecules. Curr Pharm Des 8: 671-693, 2002.

21. Hamacher J, Hadizamani Y, Borgmann M, Mohaupt M, Männel DN, Moehrlen U, Lucas R and Stammberger U: Cytokine-Ion channel interactions in pulmonary inflammation. Front Immunol 8: 1644, 2017.

22. Ito Y, Correll K, Schiel JA, Finigan JH, Prekeris R and Mason RJ: Lung fibroblasts accelerate wound closure in human alveolar epithelial cells through hepatocyte growth factor/c-Met signaling. Am J Physiol Lung Cell Mol Physiol 307: L94-L105, 2014.

23. Lindsay CD: Novel therapeutic strategies for acute lung injury induced by lung damaging agents: The potential role of growth factors as treatment options. Hum Exp Toxicol 30: 701-724, 2011.

24. Du Z, Zhang S, Lin Y, Zhou L, Wang Y, Yan G, Zhang M, Wang M, Li J, Tong Q, et al: Momordicoside G Regulates macrophage phenotypes to stimulate efficient repair of lung injury and prevent urethane-induced lung carcinoma lesions. Front Pharmacol 10: 321, 2019.

25. Monney L, Sabatos CA, Gaglia JL, Ryu A, WaldnerH, Chernova T, Manning S, Greenfield EA, Coyle AJ, Sobel RA, et al: Th1-specific cell surface protein Tim-3 regulates macrophage activation and severity of an autoimmune disease. Nature 415 : 536-541, 2002

26. Sabatos CA, Chakravarti S, Cha E, Schubart A, Sánchez-Fueyo A, Zheng XX, Coyle AJ, Strom TB, Freeman GJ and Kuchroo VK Interaction of Tim-3 and Tim-3 ligand regulates T helper type 1 responses and induction of peripheral tolerance. Nat Immunol 4: 1102-1110, 2003
27. Liu X, Jiang S, Zhang Q, Xu S, Bao X, Cao W, Bai J and Tang L: Tim-3 regulates Tregs' ability to resolve the inflammation and proliferation of acute lung injury by modulating macrophages polarization. Shock 50: 455-464, 2018

28. Yang X, Jiang X, Chen G, Xiao Y, Geng S, Kang C, Zhou T, Li Y, Guo X, Xiao H, et al: T cell Ig mucin-3 promotes homeostasis of sepsis by negatively regulating the TLR response. J Immunol 190: 2068-2079, 2013.

29. Xu X, Li H, Gong Y, Zheng $\mathrm{H}$ and Zhao D: Hydrogen sulfide ameliorated lipopolysaccharide-induced acute lung injury by inhibiting autophagy through PI3K/Akt/mTOR pathway in mice. Biochem Biophys Res Commun 507: 514-518, 2018.

30. Wu XT, Ansari AR, Pang XX, Li HZ, Zhang ZW, Luo Y, Arshad $\mathrm{M}$ and Song H: Visfatin plays a significant role in alleviating lipopolysaccharide-induced apoptosis and autophagy through PI3K/AKT signaling pathway during acute lung injury in mice. Arch Immunol Ther Exp (Warsz) 67: 249-261, 2019.

31. Brazil JC, Quiros M, Nusrat A and Parkos CA: Innate immune cell-epithelial crosstalk during wound repair. J Clin Invest 129: 2983-2993, 2019

32. Saraiva M, Vieira P and O'Garra A: Biology and therapeutic potential of interleukin-10. J Exp Med 217: pii: e20190418, 2019.

33. Kim SY, Joglekar MV, Hardikar AA, Phan TH, Khanal D, Tharkar P, Limantoro C, Johnson J, Kalionis B and Chrzanowski W: Placenta Stem/Stromal cell-derived extracellular vesicles for potential use in lung repair. Proteomics 19: e1800166, 2019.

This work is licensed under a Creative Commons Attribution-NonCommercial-NoDerivatives 4.0 International (CC BY-NC-ND 4.0) License. 